\title{
A preliminary description of evaluative morphology in LIS
}

\author{
Elena Fornasiero (Ca' Foscari University of Venice) \\ elena.fornasiero@unive.it
}

\begin{abstract}
This paper provides a preliminary description of evaluative morphology in Italian Sign Language improving a previous study by Petitta, Di Renzo, and Chiari (2015). The analysis of both elicited and corpus data reveals that LIS employs both manual and nonmanual articulators to convey evaluative features. Specifically, dedicated non-manual markers for each evaluative value combine with manual strategies involving the production of adjectives to convey endearment and pejorative, or morphological operations to encode diminutive and augmentative features: (i) manual sequential evaluation: the evaluative feature is conveyed through size and shape specifiers following the sign for the noun and displaying a modified articulation depending on the feature involved; (ii) manual simultaneous evaluation: the manual sign for the noun is modified in its articulation (restricted for the diminutive, enlarged for the augmentative). Overall, the morphological constructions detected fit the typological classification proposed for sign languages as languages exhibiting agglutinative morphology (Schuit 2007), while sharing the abstract properties identified for evaluative morphology in spoken languages, regardless of the different modality employed.
\end{abstract}

Keywords: Italian Sign Language, evaluative morphology, non-manual markers, simultaneous morphology

\section{Introduction}

Evaluative morphology (henceforth: EM) refers to the expression of a judgement (objective or subjective) about an object of the world through morphological processes (Grandi 2017). It implies two different perspectives (Grandi 2011, 6): (i) objective or quantitative, which includes diminutive (DIM) and augmentative (AUG) features related to the semantic primitives SMALL and BIG respectively; (ii) subjective or qualitative, which is encoded in endearment (END) and pejorative (PEJ) features representing the semantic primitives GOOD and BAD. In addition to morphological processes (affixation, compounding and reduplication), languages can employ other formal strategies to convey evaluative values: consonant or vowel symbolism, syntactic modification, tonal variation, - but these strategies cross the boundaries of morphology. To be defined as evaluative, a construction must satisfy two conditions (Grandi 2002, 52): a semantic condition, whereby it must assign a value, different from the standard one, to a concept; and a formal condition, namely, it must include the explicit expression of both the standard, through a lexically autonomous word-form recognised by the speakers of the language as an actual word, and the evaluative marker expressing one of the semantic values BIG, SMALL, GOOD, BAD. In the following example we see both the base 
form expressing the standard (gatt- 'cat') and a morpheme conveying the evaluative value (the suffix -ino assigning the diminutive).

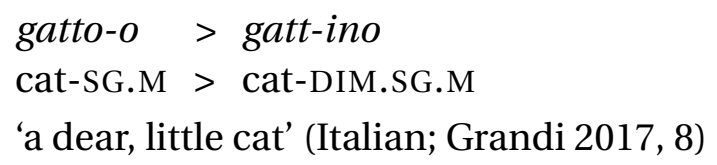

The domain of EM has been analysed from different perspectives. Numerous studies investigate its morphosyntactic nature as an inflectional or derivational process (Grandi 2017 for an overview); others focus on its morphopragmatic and semantic aspects (Dressler and Merlini-Barbaresi 1994; Jurafsky 1996). Cinque (2015) accounts for the existence of functional projections dedicated to evaluative features within the DP. Recent typological studies have defined some universal properties of EM detected cross-linguistically (Grandi and Körtvélyessy 2015; Grandi 2018): diminutive values are the most common to be encoded; therefore, if a language displays augmentatives it also displays diminutives. Moreover, the presence of qualitative evaluation (i.e. the encoding of endearment and pejorative features) implies the presence of quantitative evaluation (i.e. diminutives and augmentatives), but not vice versa. As for the morphological formal strategies mentioned above, affixation is the most common, but languages seem to display a sort of 'prefix-suffix neutrality', in that many languages can employ both prefixes and suffixes to express the same evaluative function, as shown in (2) for Italian.

\section{(2) appartament-o > appartament-ino / mini-appartament-o \\ flat-SG.M > flat-DIM.SG.M / DIM-apartment-SG.M \\ 'a small flat' (Italian; Grandi 2018, 7)}

Taking into account the morphosyntactic properties of EM, in the majority of languages it is a derivational process in that evaluative affixes: (i) typically precede inflectional ones; (ii) are not obligatory; (iii) do not carry syntactic features; (iv) can change the syntactic category of the base-word; (v) create a new lexeme with a new meaning; (vi) do not trigger agreement. However, there are some exceptions, like Bantu languages, in which EM is typically an inflectional process.

Despite the extensiveness of the studies on which the aforementioned description of the phenomenon is based, investigations only consider spoken languages. By adding sign languages (henceforth: SLs) to the cross-linguistic research, we can improve both the validity of the generalizations listed above and the types of strategies through which languages can convey the same semantic values. Therefore, the present paper aims at providing a preliminary analysis of evaluative morphology in Italian Sign Language (LIS) by describing the strategies detected and by comparing their properties to those generally associated to EM in spoken languages. The results improve the analysis provided by Petitta, Di Renzo, and Chiari (2015) who offer a panoramic description of the display of EM in several SLs. However, their study is quite general and does not consider the morphosyntactic properties of the evaluative markers involved, nor does it specify how data have been collected. Building on Petitta, Di Renzo, and Chiari (2015), the present paper provides the first results of an ongoing research whose final aim is to develop a comprehensive description and analysis of evaluative constructions in LIS. Specifically, it focuses on the morphological processes employed to convey the prototypical meanings associated to evaluative features, namely 'small', 'big' (for the objective features DIM and AUG), 'nice/lovely' and 'bad/ugly' (for subjective features END and PEJ). 
Nonetheless, I acknowledge the semantic polyvalency of evaluatives and I will investigate the possible combinations in LIS in future researches. ${ }^{1}$

The paper is organised as follows: Section 2 introduces the study of EM in SLs through the panoramic description provided by Petitta, Di Renzo, and Chiari (2015); in Section 3 the data employed for the analysis as well as the protocol of annotation are presented; Section 4 outlines the evaluative strategies detected (Section 4.1 describes manual sequential evaluation, Section 4.2 manual simultaneous evaluation, Section 4.3 non-manual simultaneous evaluation). Section 5 contains a discussion, specifically of morphological structure of nonmanual evaluation in Section 5.1, the issue of size and shape specifiers in Section 5.2, and the properties of LIS evaluative strategies in a typological perspective in Section 5.3. In Section 6 some conclusions are drawn.

\section{Evaluative morphology in sign languages}

Morphological processes in SLs can be sequential or simultaneous, displaying very different properties (Aronoff, Meir, and Sandler 2005). Sequential operations concern addition of a phonological segment to the base sign; they are rare and used for derivational processes. On the other hand, simultaneous operations are preferred and concern modification of one or more formational parameters of the manual sign (also called 'simultaneous affixation' (Pfau, Steinbach, and Woll 2012, 104), or addition of specific non-manual markers (NMMs, suprasegmental affixation). Simultaneous operations can be used for both derivational and inflectional morphology.

The preference for simultaneous operations has been related to the visuo-spatial modality (Meier, Cormier, and Quinto-Pozos 2002). Despite this, SLs are claimed to be languages with agglutinative morphology (Schuit 2007), in which all the morphemes remain identifiable and segmentable. According to the preliminary description provided by Petitta, Di Renzo, and Chiari (2015), SLs exploit both strategies to convey evaluative features. Specifically, they detect four main morphological strategies employed cross-linguistically to convey augmentation and diminution (of quantity and quality) and intensification: (i) manual sequential evaluation; (ii) manual simultaneous evaluation; (iii) non-manual simultaneous evaluation and (iv) reduplicative evaluation.

(i) manual sequential evaluation consists of the production of a canonical sign followed by a size and shape specifier (SASS) or handling classifier. According to the authors, the classifier following the canonical sign is a bound morpheme functioning as an affix. The same affix can attach to "different sign bases that share some features, depending on the properties of the referent" (Petitta, Di Renzo, and Chiari 2015, 159). They illustrate this construction with example (3).

\footnotetext{
${ }^{1}$ The same evaluative value can encode several meanings. For instance, in Italian diminutive markers can be associated to both the meanings 'small' and 'dear/nice' (see Dressler and Merlini-Barbaresi (1994) for an overview).
} 
(3) LIS (based on Petitta, Di Renzo, and Chiari 2015, 159)

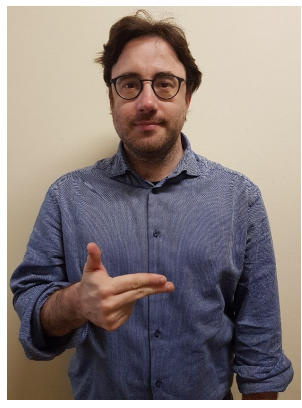

FISH

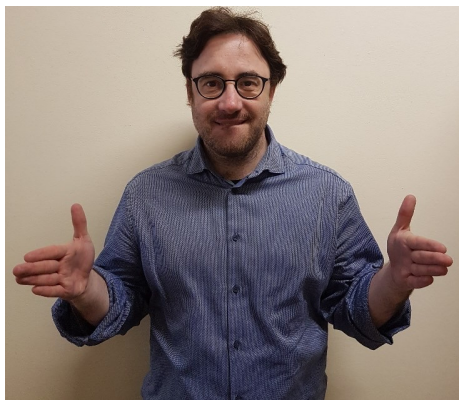

SASS:size-BIG

'a big fish'

(ii) manual simultaneous evaluation consists of the modification of the phonological features of the manual sign, which can display changes in movement, handshape or location. Specifically, augmentation and diminution can be conveyed by enlarging or reducing the handshape of the sign (examples in (4)), the distance between hands, or the movement of the sign. Morphological modifications ${ }^{2}$ are also accompanied by specific NMMs; see below.

(4) LIS (based on Petitta, Di Renzo, and Chiari 2015, 160)

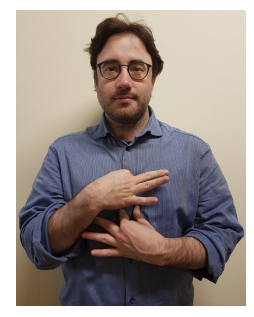

a. TIE

'a tie'

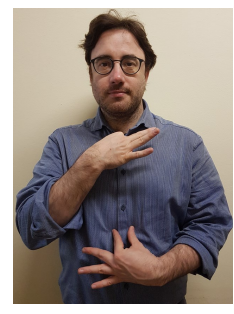

b. TIE:aug
'a big tie'
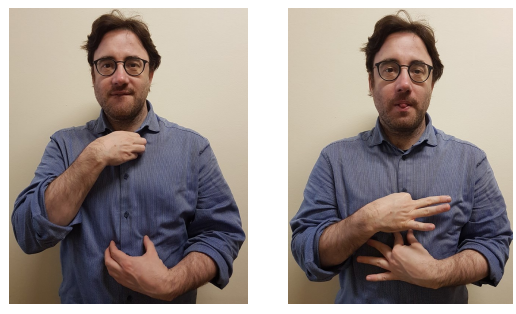

dim

c. TIE:dim

'a small tie'

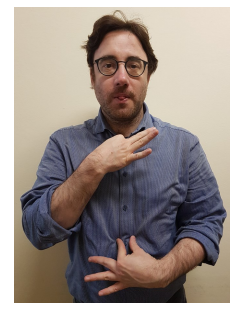

(iii) non-manual simultaneous evaluation consists of the articulation of specific NMMs modifying the manual sign articulated in its canonical form. Petitta, Di Renzo, and Chiari (2015) identify several facial expressions associated to diminutives and augmentatives in LIS. Particularly, mouth protrusion, sucked cheeks and half-protruding tongue convey the meaning 'small' (5), while puffed cheeks, teeth on lip, slightly grinding teeth and half-frown mouth convey the meaning 'big' (Petitta, Di Renzo, and Chiari 2015, 163).

\footnotetext{
${ }^{2}$ To annotate morphological modifications of the manual sign I will use the following notation: SIGN:evaluative-feature. To illustrate, TIE:aug in (4b) means that augmentative features are conveyed through a modified articulation of the manual sign.
} 
(5) LIS (based on Petitta, Di Renzo, and Chiari 2015, 164)

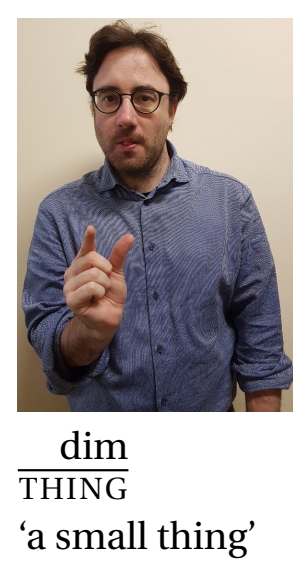

(iv) reduplicative evaluation consists of the partial or full reduplication of the base sign. Specifically, in order to convey augmentative features the copied portion following the base sign can display an enlarged articulation, as shown in example (6).

(6) LIS (based on Petitta, Di Renzo, and Chiari 2015, 166)

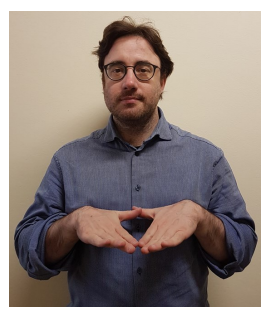

TABLE

'a big table'

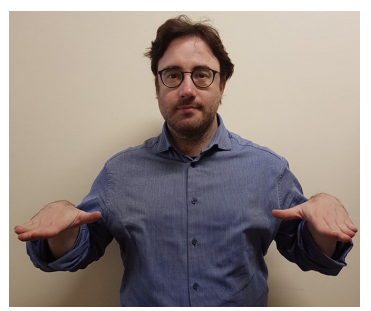

aug

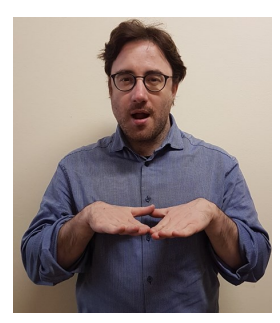

TABLE:aug

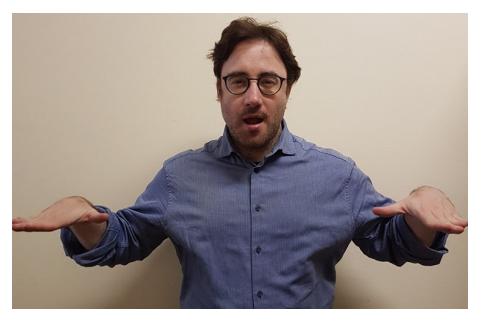

\section{Data}

In order to collect a considerable amount of data to improve the description of EM in LIS, I have analysed both naturalistic and elicited data. The analysis of naturalistic data has been useful to attest the presence of evaluative strategies in LIS. Specifically, I have analysed a corpus of 22 fairy tales ${ }^{3}$ produced by LIS native signers. Elicited data have been collected through three different tasks: object-description, narration and grammaticality judgements. The informants involved are three LIS native signers ${ }^{4}$ (mean age 38); they are all LIS teachers and have participated in linguistic research before. For the object-description task, I used drawings of objects (Figure 1a) and asked the participants to describe them; for the narration task, I asked informants to tell a story based on a series of pictures I previously showed them (Figure $1 \mathrm{~b}$ ). The items that signers were asked to describe were characterised by specific features of size, shape and quality in order to elicit both objective (diminutive, augmentative) and subjective (endearment, pejorative) descriptions. As for grammaticality judgments, I showed the informants some stills extracted from the fairy-tale corpus, which were examples of evaluative strategies, and asked them what, according to them, was the meaning of

\footnotetext{
${ }^{3} 20$ fairy tales belong to the collection 'Fiabe nel Bosco' produced by Cooperativa ALBA (Turin).

${ }^{4}$ One of them was also one of the native signers telling a fairy tale in the corpus.
} 
the sign and if they agreed on the use of that specific evaluative strategy to convey the feature. For reasons of space, in the following I will not discuss the results of the grammaticality judgment task.

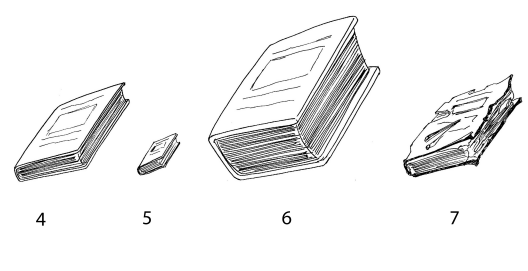

(a) Some items of the objects-description task.

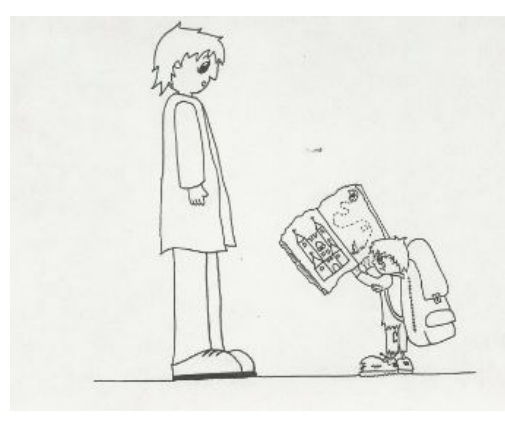

(b) Excerpt of the story for the narration task.

Figure 1: Illustrations of the elicitation stimuli.

For the analysis of both corpus and elicited data I considered: (i) nouns displaying a modified articulation (with respect to the canonical form); (ii) nouns followed by SASS; (iii) nouns followed by adjectives related to the semantic primitives BIG, SMALL, GOOD and BAD. Each occurrence was annotated and specified for the NMMs involved to convey the evaluative feature. In Table 1, I list the non-manual articulators considered and the values I annotated for each.

\begin{tabular}{l|l} 
Non-manual articulator & Values \\
\hline Eyebrows & - Furrowed \\
& - Raised \\
& - Inner brow raise (Ekman, Friesen, and Hager 2002) \\
\hline Eyes & - Open \\
& - Squinted \\
\hline Lips & - Protruded \\
& - Teeth biting the lower lip \\
\hline Mouth & - Open \\
& - Corners up \\
& - Corners down and tongue protrusion \\
& - Tongue protrusion \\
\hline Cheecks & - Inflated \\
& - Sucked-in \\
\hline
\end{tabular}

Table 1: List of non-manual articulators considered for the analysis.

The data set of annotations contains 341 evaluative items, 202 of which exploited morphological operations (both sequential and simultaneous). The other constructions involved adjectives, which were mainly used to convey endearment and pejorative features. The purpose of the present study is to provide a qualitative analysis of the phenomenon; therefore, the rest of the paper will be concerned with the description of the morphological operations involved, with particular attention to the dedicated NMMs. Since evaluative features conveyed through adjectives are not strictly morphological, they have been excluded from the analysis reported in this paper. 


\section{Results}

Overall, the main strategies employed by signers to convey evaluative features in LIS are: manual sequential evaluation and manual simultaneous evaluation, which are both characterised by the articulation of specific NMMs dedicated to the different evaluative values. The two strategies detected are mainly employed to convey augmentative and diminutive features. As mentioned above, signers mostly used adjectives to express endearment and pejorative, but I also detected some instances of non-manual simultaneous evaluation that are worth considering. I will describe and provide examples for each strategy below.

\subsection{Manual sequential evaluation}

In the examples of manual sequential evaluation detected, the sign for the noun does not display any morphological modification; instead, the SASS articulation can be enlarged or restricted to convey augmentative or diminutive features respectively (7-9).
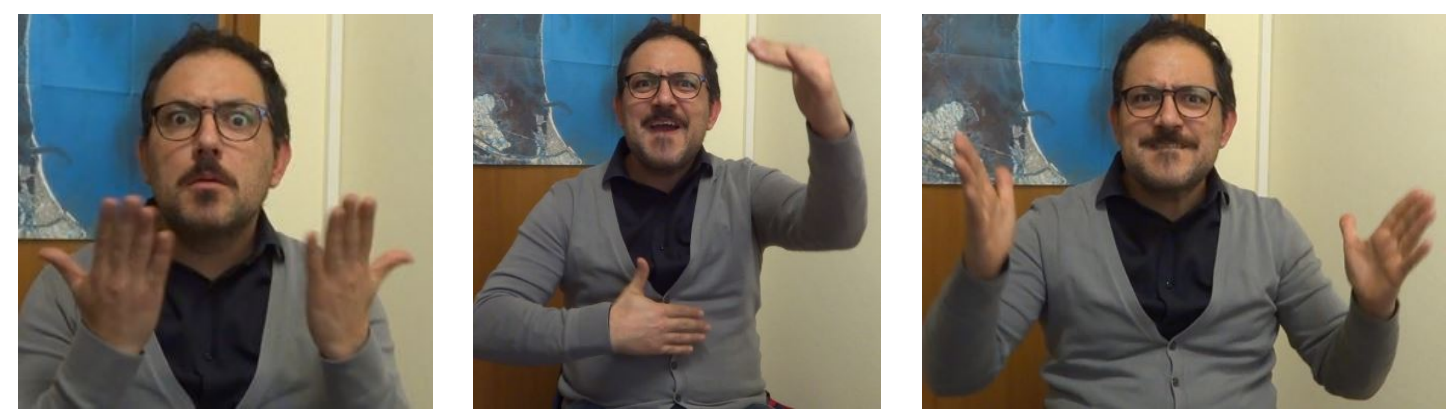

(7) $\frac{\text { aug }}{\text { BOOK SASS:shape-RECTANGULAR-BIG:aug }{ }^{5}}$
'a big rectangular book'
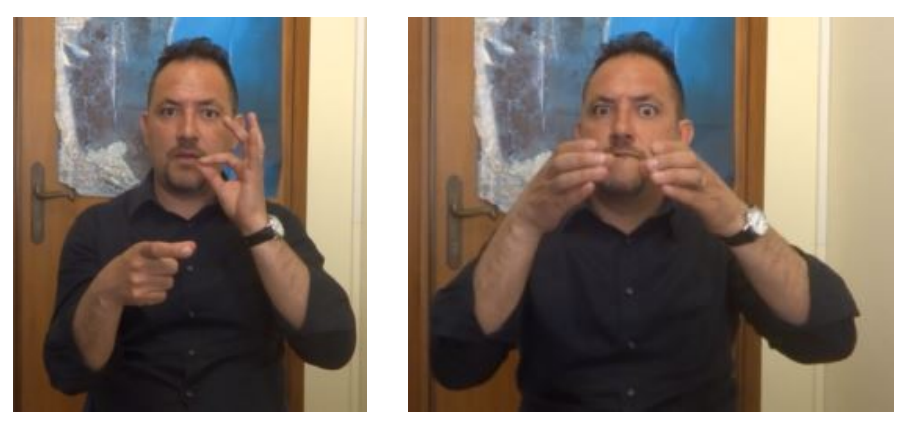

(8)

$$
\begin{aligned}
& \text { CUP SASs:shape-BIG:aug } \\
& \text { 'a big cup' }
\end{aligned}
$$

\footnotetext{
${ }^{5}$ The NMM 'wide open eyes' was mainly employed by this signer to convey the surprise of the main character of the story in finding new objects. Therefore, I do not consider it as an evaluative marker.
} 

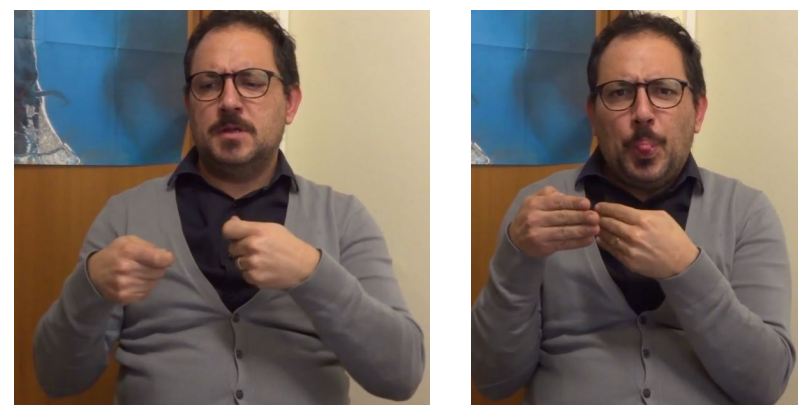

(9) SHOE SASS:size-SMALL:dim

'a little shoe'

Moreover, as we can see from the examples the SASS following the base noun are marked by specific NMMs depending on the evaluative value they are conveying: furrowed eyebrows/open eyes and teeth biting the lower lip to express augmentative features, and furrowed eyebrows, squinted eyes and tongue protrusion to convey diminutive features. ${ }^{6}$

\subsection{Manual simultaneous evaluation}

In the data, this evaluative strategy consists of the modification of one phonological segment of the manual sign, specifically the distance between hands, together with the articulation of the NMMs dedicated to diminutive or augmentative features listed above (see examples 10-13).

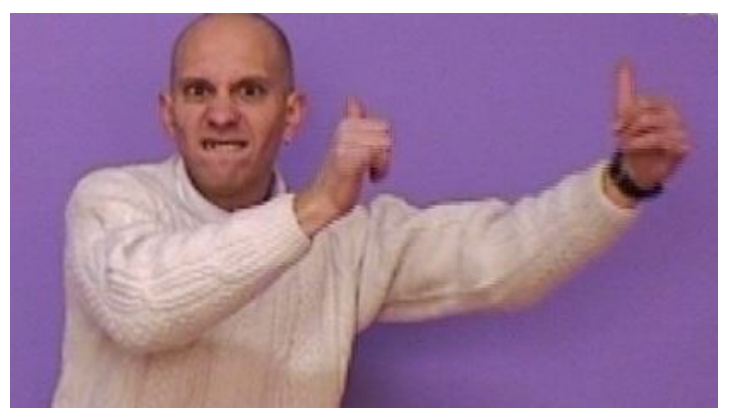

(10)

$\frac{\text { aug }}{\text { CHAIR:aug }}$
'a big chair'
(Fiabe nel Bosco, 'Riccioli d'oro')
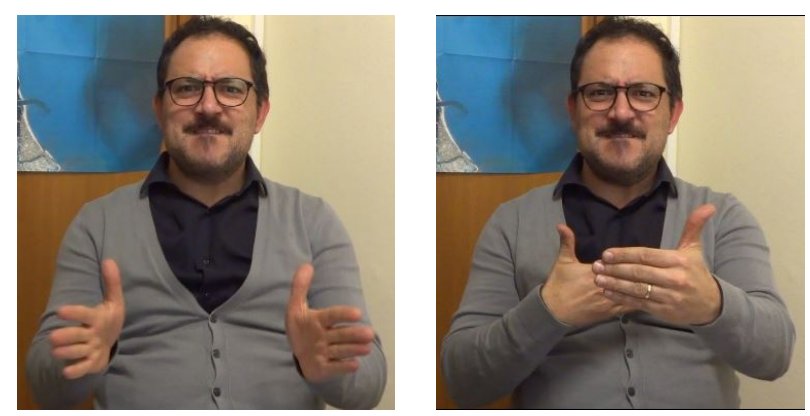

(11) $\frac{\text { aug }}{\text { BOX:aug }}$

'a big box'

\footnotetext{
${ }^{6}$ As noticed by Petitta, Di Renzo, and Chiari (2015), variation in the values of eyes (wide open or squinted) is context dependent, therefore I follow the authors in considering mouth patterns as the principal non-manual articulators related to diminutive and augmentative features.
} 


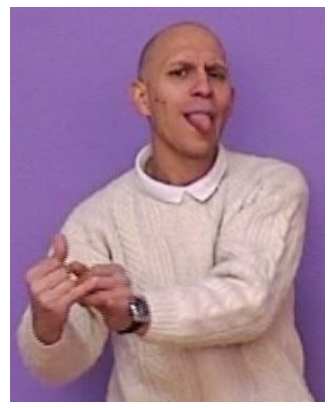

(12)

CHAIR:dim

'a little chair'

(Fiabe nel Bosco, 'Riccioli d'oro')
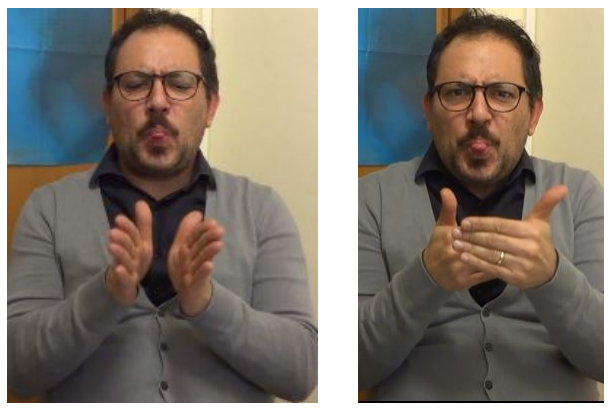

(13) $\frac{\text { dim }}{\text { Box:dim }}$

'a little box'

\subsection{Non-manual simultaneous evaluation}

Evaluative features can also be conveyed solely through specific NMMs simultaneously articulated to the manual sign, which remains in its canonical form. Even though I detected few occurrences of this strategy, examples of non-manual simultaneous evaluations are worthy to be mentioned.

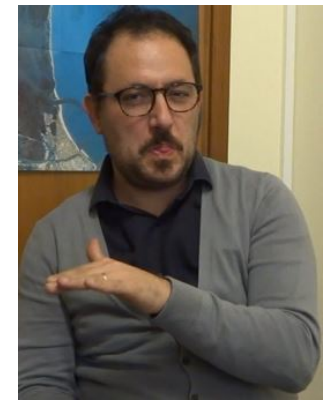

(14) $\frac{\operatorname{dim}}{\text { CHILD }}$

'a little child'

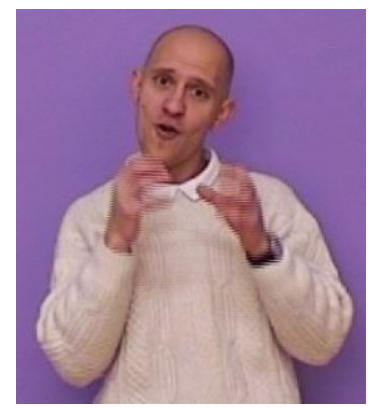

(15) $\frac{\text { end }}{\text { BEAR }}$

'a nice bear'

(Fiabe nel Bosco, 'Riccioli d'oro')

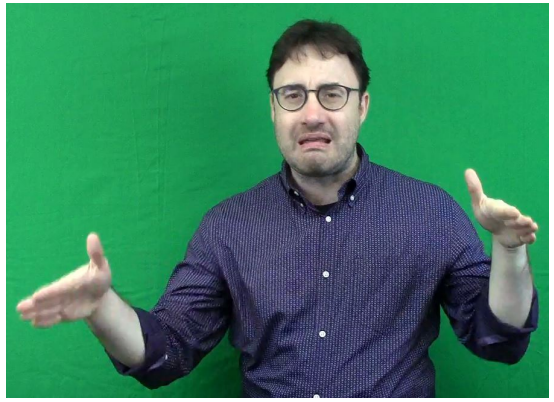

(16) $\frac{\text { pej }}{\mathrm{ROOF}}$

'a broken roof'

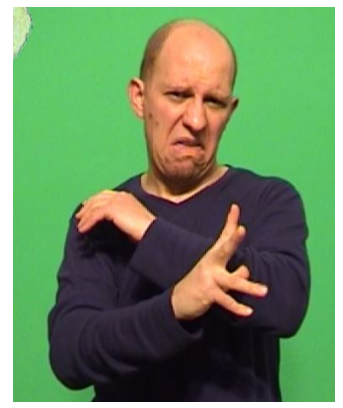

(17) $\frac{\text { pej }}{\text { GREEN }}$

'a bad-looking green'

(Fiabe nel Bosco, 'Riccioli d'oro')

Notice that this strategy can be used to modify adjectives of colour as well, as in (17). 


\section{Discussion}

\subsection{Morphological structure of NMMs}

The analysis of the data reveals that despite the different manual strategies involved, each evaluative value is specified for dedicated NMMs: (i) diminutive is conveyed through squinted eyes and tongue protrusion; (ii) augmentative is encoded through furrowed eyebrows and teeth biting the lower lip; (iii) endearment is associated to inner brow raise and slightly protruding lips; (iv) pejorative is conveyed through furrowed eyebrows and mouth corners down with tongue protrusion.

The articulation of diminutive and augmentative NMMs occurs with the articulation of SASS following the nominal signs (sequential evaluation) or with the articulation of the nominal sign, which displays a modified articulation when possible (simultaneous evaluation). Notice that also SASS following the nominal signs display an enlarged or restricted articulation to encode the augmentative or diminutive respectively. The presence or absence of a SASS seems to be related to the phonology of the nominal sign it follows: those that do not allow for a modification of their articulation to convey diminutive and augmentative values require a SASS which allow, instead, to encode this phonological modification to specify information of size. Indeed, these modifications are not attested in one-handed signs, ${ }^{7}$ nor to convey endearment and pejorative lacking the [size] feature. Therefore, it seems that DIM and AUG are conveyed through dedicated NMMs, whose presence calls for a phonological readjustment of the manual sign. When the phonological readjustment is not possible on the nominal sign, it is realised on the articulation of the SASS following the noun. In so doing, LIS behaves as some spoken languages displaying specific phonological modifications for morphological means, specifically when a diminutive or augmentative affix is attached to the stem. In example (18) from Slovak, the consonant [c] is palatalized to [̌c] when the diminutive affix $-k$ - is present (see Gregová 2011 for details).

$$
\begin{aligned}
& \text { palic- } a>\text { palič- } k-a \\
& \text { stick-SG.F }>\text { stick-DIM-SG.F } \\
& \text { 'a little stick' (Slovak; Gregová 2011, 334) }
\end{aligned}
$$

Other SLs display similar morphological processes to convey diminutive and augmentative features: see, among others, Schuit $(2007,43)$ for Sign Language of the Netherlands (Nederlandse Gebarentaal: NGT); Sutton-Spence and Woll (1999, 53;87) for British SL (BSL); Pfau and Quer $(2010,5)$ for German SL (Deutsche Gebaerdensprache: DGS); Tomaszewski and Farris $(2010,303)$ for Polish SL. Interestingly, these SLs employ the same morphological operations to encode the semantic primitives SMALL and BIG, but the NMMs involved are language-specific.

As for endearment and pejorative, data show that they are mainly conveyed through adjectives like DEAR, NICE, BAD, AWFUL, but I have also detected some instances of non-manual simultaneous evaluation in which NMMs are enough to convey these subjective values (examples (15-17)). This alternation requires further investigation.

Nevertheless, it is evident that NMMs play a crucial role in conveying evaluative features in LIS. It is widely accepted that non-manuals fulfil phonological, morphological, syntactic and prosodic functions in the grammar of SLs (Pfau and Quer 2010). Despite this, their linguistic status is sometimes questioned claiming that certain NMMs are gestures rather than

\footnotetext{
${ }^{7}$ Further investigations are necessary to test whether example (14) above constitutes an exception or it is related to other phonological or semantic reasons.
} 
grammatical markers. However, linguistic and non-linguistic non-manuals display very different properties, even if they involve the same articulators (Baker-Shenk 1983; De Vos, Van der Kooij, and Crasborn 2009): (i) linguistic NMMs activate the linguistic system in the brain (a. o. Corina, Bellugi, and Reilly 1999); (ii) they select a narrower and defined subset of facial muscles; (iii) they show clear and rapid onset and offsets corresponding to precise scope (lexical items, edge marking or domain marking (Baker-Shenk 1983); and (iv) they display a lesser degree of variation among signers. Moreover, linguistic facial markers can be grouped considering their function: it has been proposed that the articulators belonging to the lower half of the face (namely mouth, tongue, cheeks, jaws) carry adverbial and adjectival information, whereas the NMMs belonging to the upper part (eyebrows, head position, head nods, eye-gaze) are typically involved in syntactic functions (Wilbur 2000).

Considering these properties, the NMMs detected as evaluative markers are linguistic rather than gestural elements in that: (i) they consist of a selected subset of facial expressions; (ii) they display clear onset and offset corresponding to the articulation of lexical items, and (iii) they are used consistently by the different signers. As for their linguistic nature, it can be argued that they are evaluative affixes because: (i) they cannot occur alone, rather, they are bound to the manual sign with which they are simultaneously articulated; (ii) they are discrete and listable, in that specific NMMs are devoted to each evaluative feature; (iii) they carry a precise meaning modifying the semantics of the stem, i.e. the manual sign, thus playing a modification function.

If the status of NMMs in LIS evaluative constructions seem to be quite clear, we cannot claim the same for SASS, whose nature and function will be investigated in the next section.

\subsection{Size and shape specifiers}

In the data, SASS are often employed to convey diminutive and augmentative features. However, the nature and function of these elements are quite unclear in the SL literature. It is generally assumed that SASS belong to the domain of SL classifiers (a.o. Supalla 1982; EngbergPedersen 1993; Zwitserlood 2003), but they display very different properties in comparison to classifiers belonging to different categories. First of all, SASS do not combine with verbal roots of motion and location to form predicates, they rather appear in nominal constructions conveying information about the size and shape of referents. Despite this, some authors use the term 'SASS' to refer to entity classifiers employed to convey the shape of the referent. But entity classifiers and SASS, at least in LIS, display very different properties and select different handshapes (Fornasiero 2018). In the present research, I use the term 'SASS' to refer to morphologically complex elements which appear in DPs specifying information of size and shape of the referent. I provide an example below coming from a parallel study investigating the nature of SASS in LIS (Fornasiero 2018). 

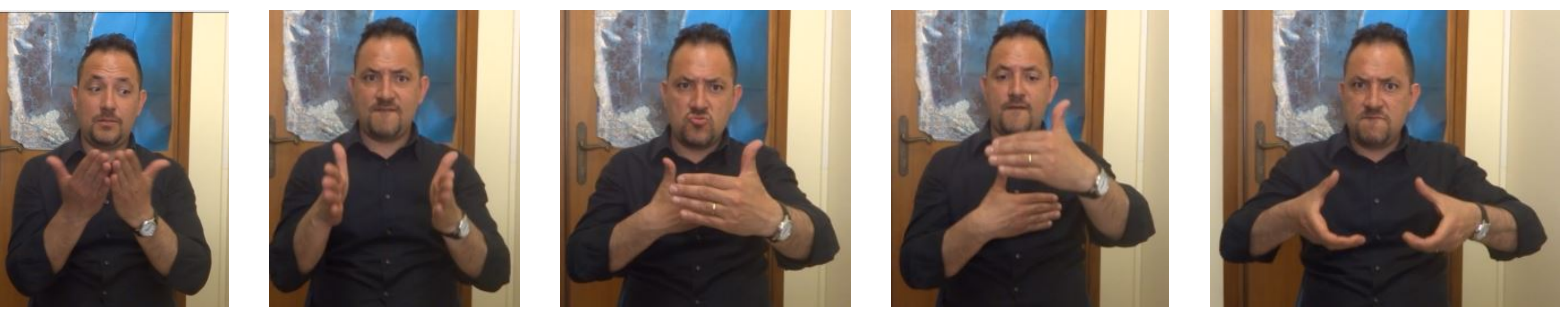

(19)

BOOK SASS:shape-RECTANGULAR:aug SASS:depth-THICK:aug SASS:size-BIG:aug 'a big thick rectangular book'

In (19), the nominal sign is followed by three different SASS defining the shape, thickness and size of the book. In the data set of LIS evaluative strategies, I have detected many instances of these elements (not necessarily all three in a row) following the nominal signs to convey diminutive and augmentative features. In these constructions, SASS undergo the same morphological modifications detected in the examples of manual simultaneous evaluation, namely modification of the articulation of the manual sign and articulation of dedicated NMMs, as also shown in (20).
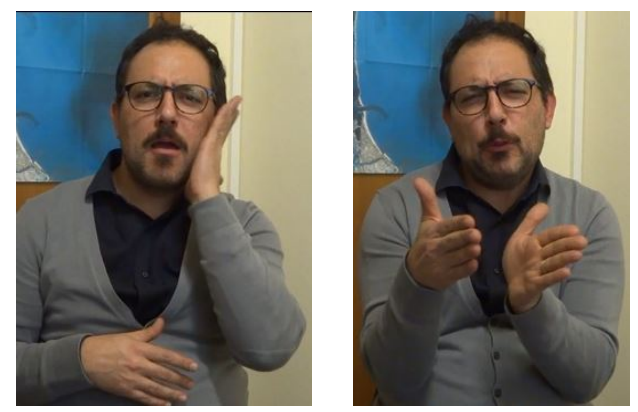

(20)

$\operatorname{dim}$

BED SASS:size-SMALL:dim

'a small bed'

Petitta, Di Renzo, and Chiari (2015) assume that SASS in these constructions are bound to the manual sign with which they occur. However, their status of affixes is not straightforward. The occurrence of SASS in nominal domains has been described by Newport and Bellugi (1978), who consider them a part of compounds in which they stand for a general class of shapes. The authors point out that the SASS in these constructions do not vary according to the details of size and shape of the referent. For instance, a brick, a postcard and a credit card will be all defined using the SASS for 'rectangular' even though they have different sizes in the real word. But this does not seem to be the case for the SASS detected in LIS to convey evaluative features.

First, the SASS I detected do not display a unique form for all the referents they appear with, rather their articulation is restricted or enlarged in order to convey diminutive and augmentative features, and their use depends on the referent they denote. Moreover, from a parallel study investigating the occurrence of these elements in DPs (Fornasiero 2018), it results that they can be separated from their lexical head, as we can see in (21) below in which the SASS specifying the shape of the hat is separated from its base noun (HAT) by the adjective of origin (MEXICAN). 

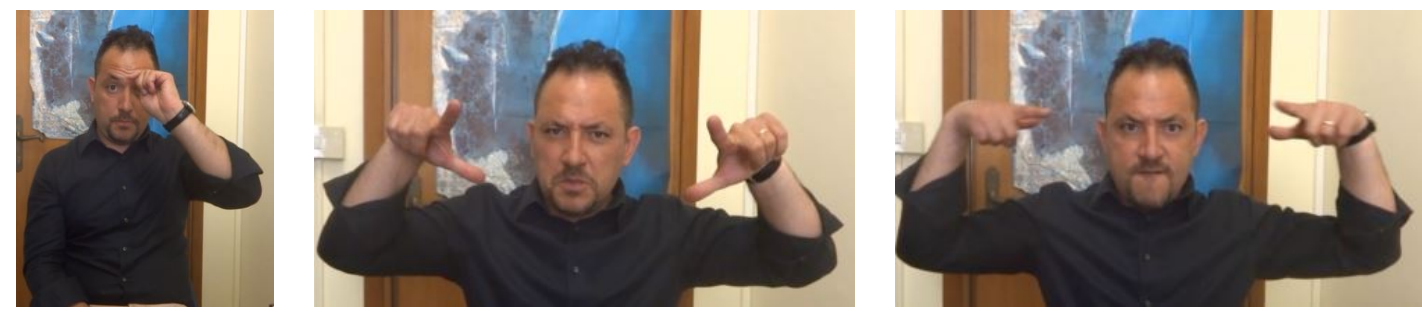

(21) HAT MEXICAN SASS:shape-ROUND:aug

'a large round Mexican hat'

Therefore, it can be reasonably argued that the SASS in LIS evaluative constructions are neither affixes nor compounds, but rather free lexical items having adjectival functions, whose linguistic nature and syntactic distribution needs to be further investigated.

Considering that EM includes processes modifying the morphology of nouns to convey evaluative features, I believe it is better to exclude SASS from this domain because they are not strictly morphological, rather they could be included in the domain of syntactic modifications to convey evaluative values.

It follows that I consider the examples of manual and non-manual simultaneous evaluation as prototypical examples of EM in LIS, in that they involve simultaneous affixation to convey diminutive, augmentative, endearment and pejorative features.

\subsection{Typological classification of evaluative morphology in LIS}

To include sign languages within typological studies investigating EM is crucial, as it allows to improve our understanding of the properties displayed and to add language-specific morphological processes, thus verifying the validity of the generalizations shaped on spoken languages.

If we take into account the examples of manual and non-manual simultaneous evaluation detected in LIS, at this stage of the research it seems that LIS respects the universals defined for EM in spoken languages as: (i) it displays evaluative features (both qualitative and quantitative); (ii) affixation is the preferred strategy (no examples of reduplication or compounding attested), although numerous examples of adjectival expression have been attested as well; (iii) evaluative constructions respect both the semantic and formal conditions defined by Grandi (2002): the manual sign represents the standard value, whereas the NMMs encode the evaluative meaning, thus conveying a new semantic concept. Moreover, LIS displays the same phonological modifications for morphological means attested in some spoken languages for diminutive and augmentative, while exploiting the unique sign language possibility of having the phonological readjustment and affixation as simultaneous rather than sequential processes, since they are realized by distinct articulators (manual and non-manual).

As for the inflectional/derivational issue, at this stage the evaluative strategies detected in LIS seem to belong to the domain of derivational morphology. Indeed, the evaluative affixes detected are not relevant to syntax, therefore they are not obligatory, nor do they convey any syntactic feature. Instead, they carry specific meanings and they derive new lexemes from existing ones. The evaluative strategies detected in LIS fall within the general assumption that SL morphology is agglutinative (Schuit 2007, 58): despite being simultaneous and suprasegmental, evaluative morphemes are identifiable and segmentable. 


\section{Conclusions}

The present paper provides a preliminary description of the display of evaluative morphology in LIS, building on and improving a previous work developed by Petitta, Di Renzo, and Chiari (2015). The analysis conducted considers both naturalistic and elicited data, which have been useful to detect the most common evaluative strategies employed and the NMMs dedicated to each evaluative feature. It shows that EM is a special kind of simultaneous morphology in that dedicated NMMs combine with segmental modifications of manual signs to convey evaluative features. The NMMs detected are evaluative affixes bound to the manual sign with which they co-occur. While the co-occurrence of manual and non-manual components to convey EM is a common strategy in SLs, the NMMs involved are sign language specific. The present analysis enriches the typological studies on EM by showing that LIS respects the generalisations proposed on the basis of the properties observed in spoken languages, despite the different modality employed.

The preliminary results presented in this study prepare the path for further investigations both at the descriptive and theoretical level. More data is needed to confirm and improve the findings. Specifically, we need to find out: (i) whether the NMMs here identified as evaluative are, instead, phonological features of the manual adjectives BIG, SMALL, GOOD, BAD: if this was the case, it could be possible that the NMMs are actually non-manual adjectives whose manual part has been dropped; (ii) the possibility of applying evaluative strategies to abstract nouns; (iii) the phonological restrictions calling for the presence of SASS to convey evaluative features. Moreover, further investigation is needed to account for the nature and distribution of SASS as bound affixes or, rather, as free lexical items in order to develop a unified morphological model able to account for the different strategies detected.

\section{Acknowledgments}

This work is funded by the SIGN-HUB project (European Union's Horizon 2020 Research and Innovation Program, Grant Agreement 693349). Many thanks to the LIS informants for sharing their language, to Claudio and Cooperativa ALBA for allowing me to reproduce some extracts of the fairy tales corpus Fiabe nel Bosco, and to the audience of FEAST 2018 in Venice for their valuable comments.

\section{References}

Aronoff, Mark, Irit Meir, and Wendy Sandler. 2005. "The paradox of sign language morphology". Language 81 (2): 301-344.

Baker-Shenk, Charlotte Lee. 1983. "A microanalysis of the non-manual components of questions in American Sign Language”. Doctoral dissertation, University of California.

Cinque, Guglielmo. 2015. "Augmentative, pejorative, diminutive and endearing heads in the extended nominal projection”. In Structures, strategies and beyond: Studies in honour of Adriana Belletti, ed. by Elisa Di Domenico, Cornelia Hamann, and Simona Matteini, 6782. Amsterdam: John Benjamins.

Cooperativa ALBA (Turin). Fairy tales in LIS: Fiabe nel Bosco. www . babacova . com. 
Corina, David P., Ursula Bellugi, and Judy Reilly. 1999. "Neuropsychological studies of linguistic and affective facial expressions in deaf signers". Language and Speech 42 (2-3): 307-331.

De Vos, Connie, Els Van der Kooij, and Onno Crasborn. 2009. "Mixed signals. Combining linguistic and affective functions of eye brows in questions in Sign Language of the Netherlands". Language and Speech 52 (2): 315-339.

Dressler, Wolfgang U., and Lavinia Merlini-Barbaresi. 1994. Morphopragmatics: Diminutives and intensifiers in Italian, German, and Other Languages. Berlin: De Gruyter Mouton.

Ekman, Paul, Wallace V. Friesen, and Joseph C. Hager. 2002. Facial Action Coding System. Salt Lake City.

Engberg-Pedersen, Elisabeth. 1993. Space in Danish Sign Language: The semantics and morphosyntax of the use of space in a visual language. Hamburg: Signum.

Fornasiero, Elena. 2018. "A morphological analysis of size and shape specifiers in Italian Sign Language”. PhD seminar, Ca’ Foscari University of Venice.

Grandi, Nicola. 2002. Morfologie in contatto. Le costruzioni valutative nelle lingue del Mediterraneo. Milan: Franco Angeli.

- .2011. "Renewal and innovation in the emergence of Indo-European evaluative morphology”. Lexis 6 (). doi:10.4000/lexis . 403.

— . 2017. "Evaluatives in morphology". In Oxford research encyclopedia of linguistics, 1-21. Oxford: Oxford University Press.

— . 2018. "Typological tendencies in evaluative morphology". In Word-Formation Theories III \& Typology and Universals in Word-Formation IV. Kosice.

Grandi, Nicola, and Livia Körtvélyessy. 2015. The Edinburgh handbook of evaluative morphology. Edinburgh: Edinburgh University Press.

Gregová, Renata. 2011. "Evaluative morphology and (mor)phonological changes in diminutives of Indo-European languages". BAS, British and American Studies, A Journal of The Romanian Society of English and American Studies 17:333-343.

Jurafsky, Daniel. 1996. "Universal tendencies in the semantics of the diminutive". Language 72 (3): 533-578.

Meier, Richard P., Kearsey Cormier, and David Quinto-Pozos. 2002. Modality in signed and spoken languages. Cambridge: Cambridge University Press.

Newport, Elissa, and Ursula Bellugi. 1978. "Linguistic expression of category levels in a visualgestural language: a flower is a flower is a flower". In Cognition and categorisation, ed. by Eleanor Rosch and Barbara Lloyd. Hillsdale, NJ: Lawrence Erlbaum Associates.

Petitta, Giulia, Alessio Di Renzo, and Isabella Chiari. 2015. "Evaluative morphology in sign languages". In Edinburgh handbook of evaluative morphology, ed. by Nicola Grandi and Livia Körtvélyessy, 155-169. Edinburgh: Edinburgh University Press.

Pfau, Roland, and Josep Quer. 2010. "Nonmanuals: their grammatical and prosodic roles". In Sign Languages, ed. by Diane Brentari, 381-402. Cambridge: Cambridge University Press.

Pfau, Roland, Markus Steinbach, and Bencie Woll, eds. 2012. Sign language. An international handbook. Berlin: De Gruyter Mouton.

Schuit, Joke. 2007. "The typological classification of sign language morphology". MA thesis, University of Amsterdam. 
Supalla, Ted. 1982. "Structure and acquisition of verbs of motion and location in American Sign Language." PhD thesis, University of California.

Sutton-Spence, Rachel, and Bencie Woll. 1999. The linguistics of British Sign Language. Cambridge: Cambridge University Press.

Tomaszewski, Piotr, and Michael Farris. 2010. "Not by the hands alone: functions of nonmanual features in Polish Sign Language". In Studies in the psychology of language and communication, ed. by Barbara Bokus, 289-320. Warszawa: Matrix.

Wilbur, Ronnie B. 2000. "Phonological and prosodic layering of nonmanuals in American Sign Language". In The signs of language revisited. An anthology to honor Ursula Bellugi and Edward Klima, ed. by Karen Emmorey and Harlan L. Lane, 215-244. Mahwah, NJ: Lawrence Erlbaum Associates.

Zwitserlood, Inge. 2003. “Classifying hand configurations in Nederlandse Gebarentaal”. Doctoral dissertation, LOT. 